\title{
The University of Chicago Comprehensive Cancer Center
}

National Cancer Institute

\section{Source}

National Cancer Institute. The University of Chicago Comprehensive Cancer Center. NCI

Thesaurus. Code C39549.

The mission of the University of Chicago Comprehensive Cancer Center is to improve the understanding of the determinants of cancer, its prevention, and its treatment. Research programs emphasize advances in chemotherapy, hormonal therapy, gene therapy, and bone marrow transplantation. It was designated as an NCl cancer center in 1973 and as a comprehensive cancer center in 2008. 